\title{
A Melon Fruit Grading Machine Using a Miniature VIS/NIR Spectrometer: 2. Design Factors for Optimal Interactance Measurement Setup
}

\author{
Sang Ryong Suh, Kyeong-Hwan Lee*, Seung Hwa Yu, Hwa Sun Shin, Soo Nam Yoo, Yong Soo Choi \\ Department of Rural and Biosystems Engineering, Chonnam National University, Gwangju, Korea \\ Received: May 30 ${ }^{\text {th }}, 2012$; Revised: June 27 ${ }^{\text {th }}, 2012$; Accepted: June $29^{\text {th }}, 2012$
}

\section{Abstract}

Purpose: In near infrared spectroscopy, interactance configuration of a light source and a spectrometer probe can provide more information regarding fruit internal attributes, compared to reflectance and transmittance configuration. However, there is no through study on the parameters of interactance measurement setup. The objective of this study was to investigate the effect of the parameters on the estimation of soluble solids content (SSC) and firmness of muskmelons. Methods: Melon samples were taken from greenhouses at three different harvesting seasons. The prediction models were developed at three distances of 2, 5, and $8 \mathrm{~cm}$ between the light source and the spectrometer probe, three measurement points of 2, 3, and 6 evenly distributed on each sample, and different number of fruit samples for calibration models. The performance of the models was compared. Results: In the test at the three distances, the best results were found at a $5 \mathrm{~cm}$ distance. The coefficient of determination $\left(\mathrm{R}_{\mathrm{cv}}{ }^{2}\right)$ values of the cross-validation were 0.717 (standard error of prediction, $\mathrm{SEP}=1.16^{\circ} \mathrm{Brix}$ ) and $0.504(\mathrm{SEP}=4.31 \mathrm{~N})$ for the estimation of SSC and firmness, respectively. The minimum measurement point required to fully represent the spectral characteristics of each fruit sample was 3 . The highest $\mathrm{R}_{\mathrm{cv}}{ }^{2}$ values were $0.736\left(\mathrm{SEP}=0.87{ }^{\circ} \mathrm{Brix}\right)$ and $0.644(\mathrm{SEP}=4.16 \mathrm{~N})$ for the estimation of SSC and firmness, respectively. The performance of the models began to be saturated when 60 fruit samples were used for developing calibration models. The highest $\mathrm{R}_{\mathrm{cv}}{ }^{2}$ of 0.713 (SEP=0.88 ${ }^{\circ} \mathrm{Brix}$ ) and $0.750(\mathrm{SEP}=3.30 \mathrm{~N})$ for the estimation of SSC and firmness, respectively, were achieved. Conclusions: The performance of the prediction models was quite different according to the condition of interactance measurement setup. In designing a fruit grading machine with interactance configuration, the parameters for interactance measurement setup should be chosen carefully.

Keywords: Firmness, Interactance configuration, Muskmelon, Soluble solids content (SSC), VIS/ NIR spectroscopy

\section{Introduction}

Near infrared (NIR) spectroscopy has great potential for the estimation of internal quality of fruits in a rapid and nondestructive way. In NIR spectroscopy, a fruit is illuminated with NIR radiation and the reflected or transmitted radiation is measured by a spectrometer. The reflectance or transmittance spectra contain the characteristics of both absorption and scattering properties of the internal tissue. Absorption property is affected by the

\footnotetext{
*Comesponding author: Kyeong-Hwan Lee

Tel: +82-62-530-2156; Fax: +82-62-530-2159

E-mail: khlee@jnu.ac.kr
}

chemical composition of the fruit and scattering property is related to the texture of the fruit. Therefore, NIR spectroscopy can be used to measure both absorption-related attributes such as soluble solids content (SSC) of fruits and texture-related attributes such as firmness (Zude et al., 2006; Nicolai et al., 2007; Penchaiya et al., 2009; Sun et al., 2010).

An NIR spectroscopy system consists of a light source and a spectrometer including an optical fiber sensing probe, monochromator, detector, and beam splitter. There are three different spectra acquisition modes based on the configuration of a light source and a spectrum sensing probe: reflectance, transmittance, and interactance modes 
(Schaare and Fraser, 2000; Nicolai et al., 2007; Suh et al., 2011). In reflectance mode, a light source and a sensing probe are located at a specific angle, facing fruit surface, to avoid specular reflection. Reflectance measurements are easiest to obtain and the intensity level of the spectra is relatively high. However, the measurements mainly include superficial or surface properties of the fruit. In transmittance mode, a light source is positioned diametrically opposite to a sensing probe. Transmittance measurements are better for detecting internal damage than reflectance measurements. However, the light source with high power, which can easily burn the fruit surface and change its spectral properties, should be used to increase the amount of light penetrating the fruit. In interactance mode, a light source and a sensing probe are positioned parallel to each other so that light by specular reflection cannot enter the probe. This can be achieved by a special optical arrangement in which the light source and the probe are separated and positioned in some distance. In selecting an optimal spectra acquisition mode, it is important to consider the penetration of light into fruit tissue and its travel path. In interactance mode, the penetration depth and travel path of the light detected by the sensing probe can be various according to the arrangement between the light source and the sensing probe. The optimum arrangement can be determined based on the types of fruit, fruit surface and internal tissue characteristics, and the power consumption of light source. Owing to these characteristics, interactance measurements can include more information regarding fruit internal quality, compared to reflectance and transmittance measurements. Schaare and Fraser (2000) compared reflectance, transmittance, and interactance modes in measuring SSC, density, and internal flesh color of kiwifruit. They reported that interanctance mode showed the most accurate results.

However, there is no through study on the configuration parameters for interactance measurements. The objective of this study was to investigate the effect of the distance between a light source and a spectrum sensing probe, the minimum number of measurements on a single fruit with unevenly distributed fruit quality attributes, and the minimum number of fruit samples to develop calibration models for estimation of muskmelon SSC and firmness in interactance mode.

\section{Materials and Methods}

\section{Experimental materials}

Muskmelon (Cucumis melo L.) samples were taken from local greenhouses at Naju, Korea in three different harvesting seasons of February through March, 2009 (experiment 1), June through July, 2009 (experiment 2), and March, 2010 (experiment 3). The numbers of samples for each experiment were 93,104, and 60, respectively. The detailed properties of the fruit samples are explained in Suh et al. (2012). An interanctance spectral data acquisition system composed of a miniature VIS/NIR spectrometer (model: USB4000, Ocean Optics, FL, USA), an external light source, optical fibers, and a computer is also described in Suh et al. (2012).

\section{Experimental method}

The interactance spectra were collected at evenly distributed 6 points on an equator of each sample. The SSC and firmness of melon samples were measured by destructive methods at the 6 points where the spectral data were collected. The mean and the coefficient of variance (CV) of the 6 SSC and firmness values were calculated and used as representative constituents for each sample. In each experiment, statistical analyses on the representative constituents and the CV values for all samples were conducted.

The calibration models for the prediction of SSC and firmness were developed using the spectral data sets collected at 2, 3, and 6 points evenly distributed on an equator of each sample. The preprocessing methods that showed the best results for each experiment were applied to the data sets (Suh et al., 2012). The performance of the models developed using the data sets collected at 2,3 , and 6 points was compared. The calibration models were also developed using the various numbers of fruit samples: 20 to 90 samples with a 10 sample increment for experiment 1,20 to 100 samples with a 10 sample increment for experiment 2 , and 20 to 60 samples with a 10 sample increment for experiment 3 . The performance of the models was compared at different numbers of fruit samples.

The spectrum characteristics of fruit samples in the interactance configuration can be determined based on the levels of fruit internal attributes and the distance between the light source and the spectrum sensing probe because the penetration depth and travel path of incident light beam can be changed with the distance. To determine the optimum distance, spectral data were collected at the 
distances of 2,5 , and $8 \mathrm{~cm}$ in experiment 3 . The integration time of the spectrometer was set to 100,1000 , and $1000 \mathrm{~ms}$ for the three distances, respectively. The calibration models for the prediction of SSC and firmness were developed using the data sets collected at the three distances. The models were evaluated by the cross validation.

\section{Results and Discussion}

\section{SSC and firmness measurements}

Table 1 shows statistical analyses on the SSC and firmness measurements. The SSC and firmness for all experiments are in ranges of 4.9-14.2 ${ }^{\circ} \mathrm{Brix}$ and 6.5-42.8 N, respectively. The CV values of SSC and firmness at the 6 points are ranged in $1.5-15.0 \%$ and $2.4-27.4 \%$, respectively. This indicates that the constituent level at each point of a single fruit sample with a diameter of 11.9-17.5 $\mathrm{cm}$ can be quite different. Thus, to improve the accuracy for the prediction of SSC and firmness, spectra and fruit attribute data should be collected at as many points as possible on each fruit sample. However, the larger measurement points, the larger the number of sensing probe so that the measurement system is more complicated and the speed for grading fruits can decrease. Therefore, the minimum number of measurement points that can fully represent the characteristics of each fruit sample needs to be studied.

\section{Effect of the number of measurement points}

Figures 1 and 2 show the coefficient of determination $\left(\mathrm{R}_{\mathrm{cv}}{ }^{2}\right)$ and standard error of prediction (SEP) values of the cross validation at different measurement points for the prediction of SSC and firmness, respectively. As expected, the highest $\mathrm{R}_{\mathrm{cv}}{ }^{2}$ (the lowest SEP) is found generally in the models using the data sets collected at all 6 points. In general, the $\mathrm{R}_{\mathrm{cv}}{ }^{2}$ increases (the SEP decreases) as the number of measurement point increases. The slopes of the change of $\mathrm{R}_{\mathrm{cv}}{ }^{2}$ and SEP between the data sets of 3 measurement points and 6 measurement points are much lower than those between the data sets of 2 measurement points and 3 measurement points. This implies that the contribution of the spectral data collected at more than 3 measurement points to the prediction models is not much considerable. Therefore, it can be believed that spectra in interactance configuration for the prediction of melon internal quality need to be collected at more than 3 points on a single melon sample with a diameter of 11.9 $17.5 \mathrm{~cm}$.

In the prediction of SSC, the $\mathrm{R}_{\mathrm{cv}}{ }^{2}$ values of the models

Table 1. Statistical analyses of SSC and firmness measurements

\begin{tabular}{|c|c|c|c|c|c|c|c|c|}
\hline \multirow{3}{*}{ Exp. } & \multicolumn{4}{|c|}{ SSC } & \multicolumn{4}{|c|}{ Firmness } \\
\hline & \multicolumn{2}{|c|}{ SSC ( ${ }^{\circ}$ Brix) } & \multicolumn{2}{|c|}{ CV (\%) } & \multicolumn{2}{|c|}{ Firmness (N) } & \multicolumn{2}{|c|}{ CV (\%) } \\
\hline & Range & Mean & Range & Mean & Range & Mean & Range & Mean \\
\hline 1 & $6.0-14.1$ & 9.7 & $2.1-12.2$ & 6.1 & $6.7-36.0$ & 22.0 & $3.6-24.5$ & 10.1 \\
\hline 2 & 4.9-13.9 & 10.2 & $1.5-9.4$ & 5.6 & $13.4-42.8$ & 26.4 & $2.4-13.8$ & 6.3 \\
\hline 3 & $5.6-14.2$ & 9.8 & $3.5-15.0$ & 7.0 & $6.5-42.4$ & 20.1 & $4.3-27.4$ & 10.1 \\
\hline
\end{tabular}

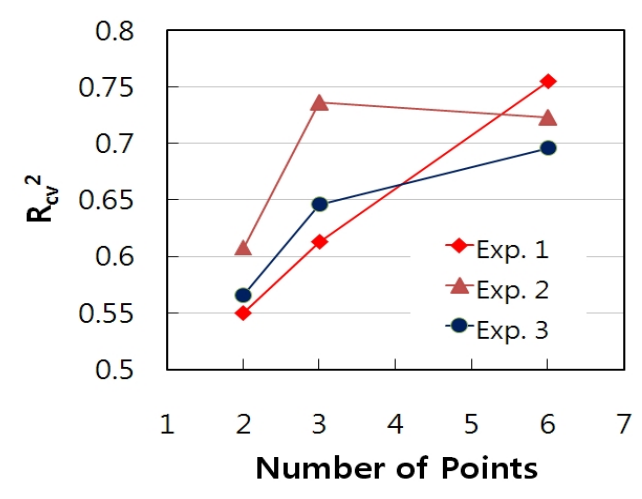

(a)

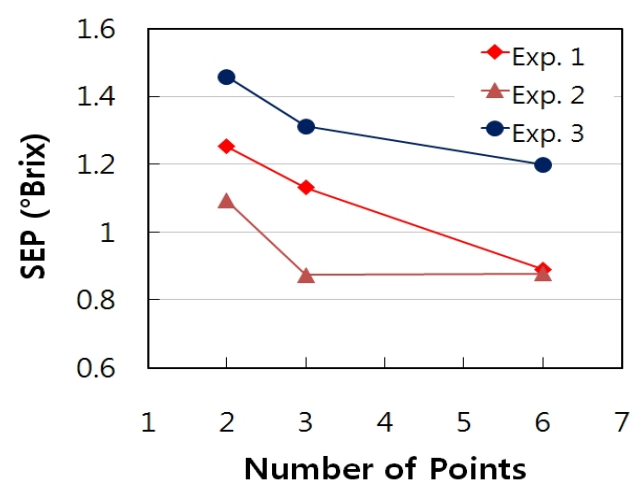

(b)

Figure 1. Performance of SSC prediction models with the data sets of different measurement points: (a) $R_{c v}{ }^{2}$ and (b) SEP. 


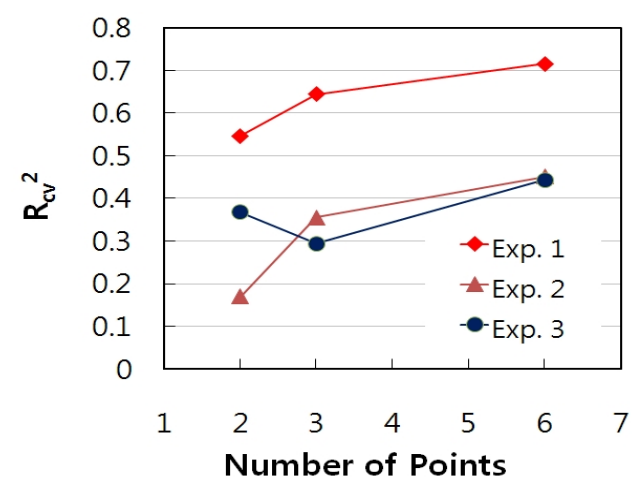

(a)

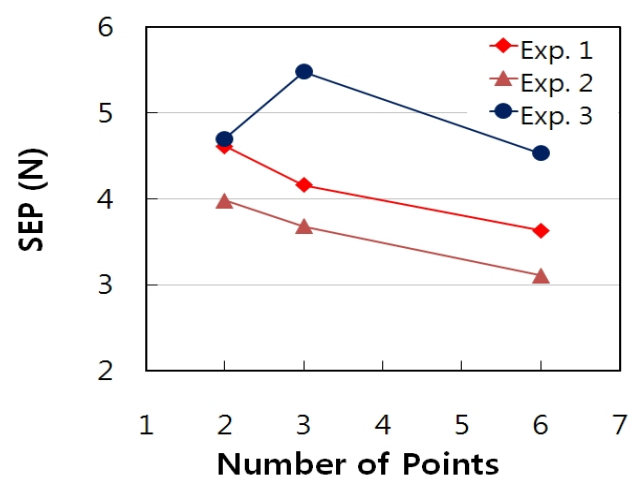

(b)

Figure 2. Performance of firmness prediction models with the data sets of different measurement points: (a) $R_{c v}{ }^{2}$ and (b) SEP.

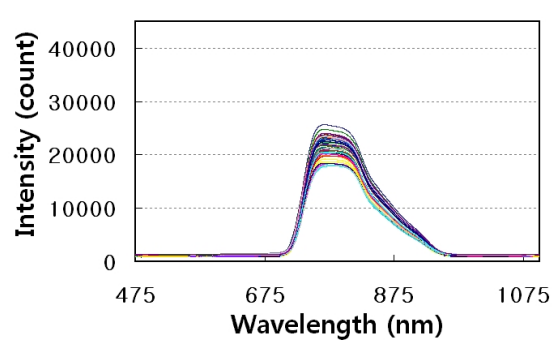

(a)

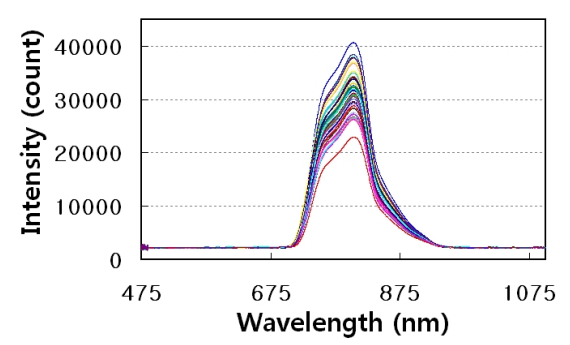

(b)

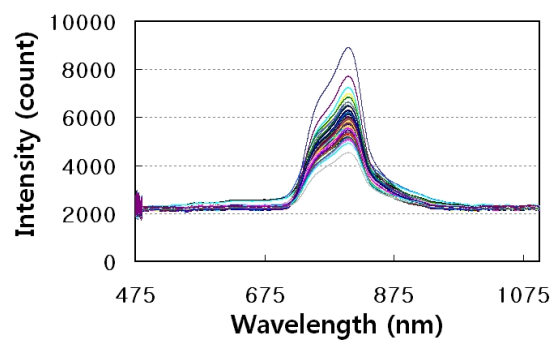

(c)

Figure 3. Intensity of the spectra in experiment 3 at the three distances: (a) $2 \mathrm{~cm}$, (b) $5 \mathrm{~cm}$, and (c) $8 \mathrm{~cm}$.

Table 2. Performance of the prediction models for the prediction of SSC and firmness at the three distances of 2, 5, $8 \mathrm{~cm}$ between the light source and the spectrometer probe in experiment 3

\begin{tabular}{|c|c|c|c|c|c|}
\hline \multirow{2}{*}{ Fruit constituent } & \multirow{2}{*}{$\begin{array}{c}\text { Distance } \\
\quad(\mathrm{cm})\end{array}$} & \multicolumn{2}{|c|}{ Calibration } & \multicolumn{2}{|c|}{ Cross Validation } \\
\hline & & Rc2 & SEC $\left({ }^{\circ}\right.$ Brix, N) & Rcv2 & SEP $\left({ }^{\circ}\right.$ Brix, N) \\
\hline \multirow{3}{*}{ SSC } & 2 & 0.793 & 0.98 & 0.696 & 1.20 \\
\hline & 5 & 0.833 & 0.88 & 0.717 & 1.16 \\
\hline & 8 & 0.952 & 0.47 & 0.707 & 1.18 \\
\hline \multirow{3}{*}{ Firmness } & 2 & 0.480 & 4.24 & 0.368 & 4.70 \\
\hline & 5 & 0.723 & 3.14 & 0.504 & 4.31 \\
\hline & 8 & 0.853 & 2.29 & 0.497 & 4.37 \\
\hline
\end{tabular}

using the data sets of 3 measurement points are 0.613 (SEP=1.13 ${ }^{\circ}$ Brix), $0.736\left(\mathrm{SEP}=0.87^{\circ} \mathrm{Brix}\right)$, and 0.646 (SEP= $1.31^{\circ}$ Brix) for experiments 1,2 , and 3, respectively. The $\mathrm{R}_{\mathrm{cv}}{ }^{2}$ values for the prediction of firmness are 0.644 (SEP= $4.16 \mathrm{~N}), 0.356(\mathrm{SEP}=3.68 \mathrm{~N})$, and $0.294(\mathrm{SEP}=5.48 \mathrm{~N})$ for experiments 1,2 , and 3 , respectively.

\section{Effect of the distance between a light source and a detector}

The spectra collected at the three distances of 2,5 , and $8 \mathrm{~cm}$ between the light source and the spectrum sensing probe are shown in Figure 3. The difference of the highest intensity levels at the three distances is caused by the different combinations of the distance, which affects the number of photons reached the sensing probe by transmitting through internal tissue from a light source, and the integration time, which determines the time for collecting the photons. The intensity level at a $5 \mathrm{~cm}$ distance with a $1000 \mathrm{~ms}$ integration time is highest compared to the intensity levels at the other distances. Also, the intensity variation with different constituent levels in a range of 750-810 $\mathrm{nm}$ is largest at a $5 \mathrm{~cm}$ distance.

Table 2 shows the performance of the models for the prediction of SSC and firmness at the three distances in 
experiment 3. The highest $\mathrm{R}_{\mathrm{cv}}{ }^{2}$ values of 0.717 (SEP $=1.16$ $\left.{ }^{\circ} \mathrm{Brix}\right)$ and $0.504(\mathrm{SEP}=4.31 \mathrm{~N})$ for the prediction of SSC and firmness, respectively, are found at a $5 \mathrm{~cm}$ distance. The diameter of fruit samples in experiment 3 was ranged in $12-15 \mathrm{~cm}$ with a mean of $13 \mathrm{~cm}$. The $5 \mathrm{~cm}$ distance that shows the best prediction result is slightly smaller than a mean radius of fruit samples. From this observation, it can be believed that the light photons detected by a spectrometer probe may transmit mainly through the area between the center of fruits and the surface of fruits where the SSC and firmness are measured by destructive methods. Therefore, it might be suggested that when a light source and a spectrometer probe are set up, the distance between them needs to be slightly smaller than a radius of fruit samples. This setup will make the amount of light photon transmitting through the interesting area of internal tissue and detected by a spectrometer greater so that the collected spectra will be more sensitive to the variation of fruit internal constituents.

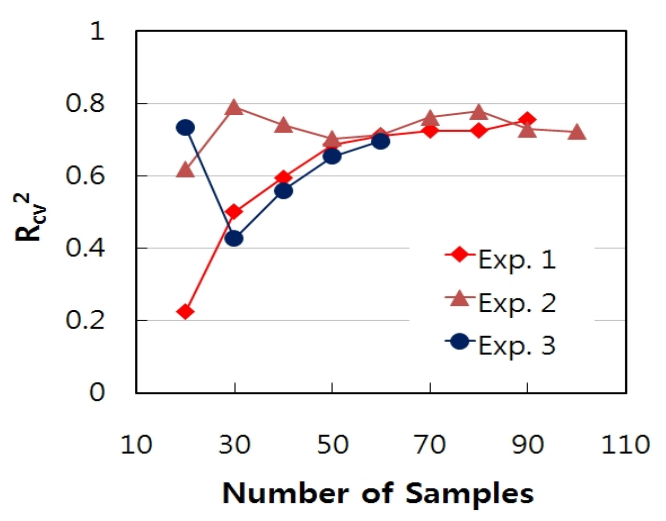

(a)

\section{Minimum number of fruit samples for calibration models}

The spectral characteristics of fruits are sensitive to within plant variability (plant age, crop load, crop position within the plant, and light effect), within greenhouse variability (position in the greenhouse, nutrition, climate, and light effect), fruit maturity, fruit cultivar, and seasonal variability (Nicolai et al., 2007; Penchaiya et al., 2009). To achieve high accuracy for the prediction of fruit attributes, the calibration models should be developed at each variability condition. However, since this calibration process is time-consuming, labor-intensive, and highlycosted, the minimum number of fruit samples that can include fruit variability as much as possible should be chosen for the calibration process.

The calibration models for the prediction of SSC and firmness were built using the various numbers of fruit samples in experiments 1,2 , and 3 . The $\mathrm{R}_{\mathrm{cv}}{ }^{2}$ and SEP values of the models are shown in Figures 4 and 5 for the

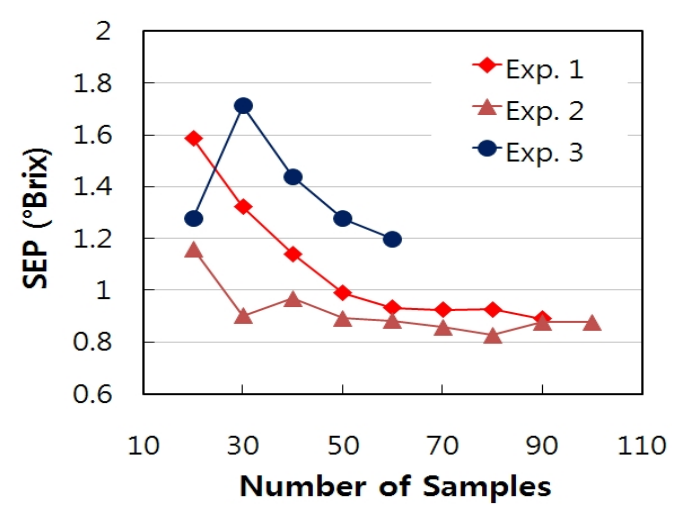

(b)

Figure 4. Performance of the SSC prediction models with the data sets of different numbers of samples: (a) $R_{c v}{ }^{2}$ and (b) SEP.

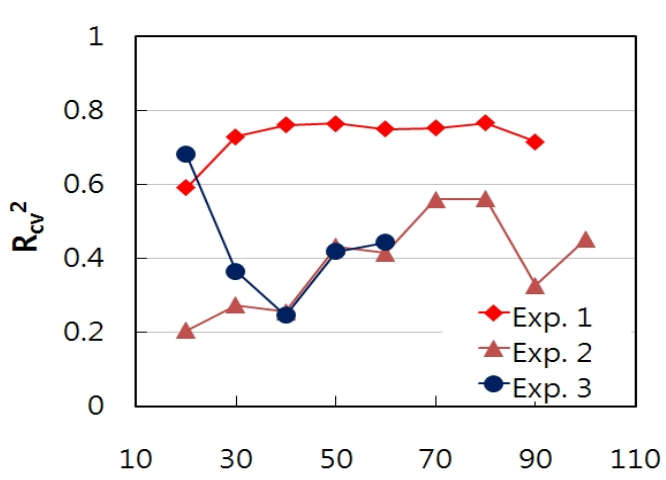

Number of Samples

(a)

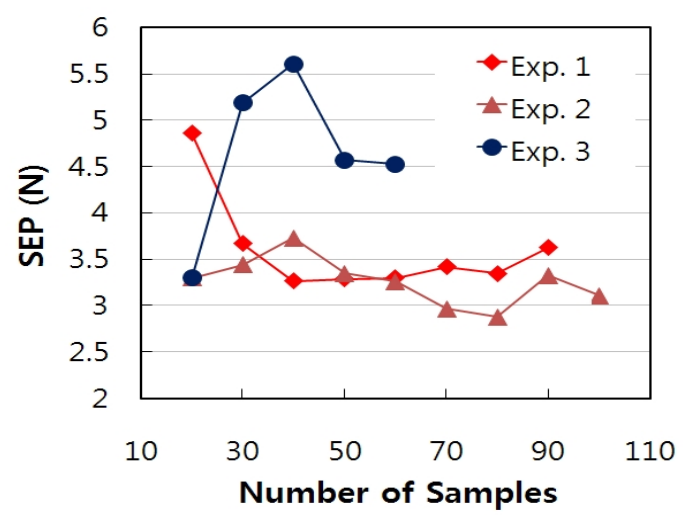

(b)

Figure 5. Performance of the firmness prediction models with the data sets of different numbers of samples: (a) $R_{c V}{ }^{2}$ and (b) SEP. 


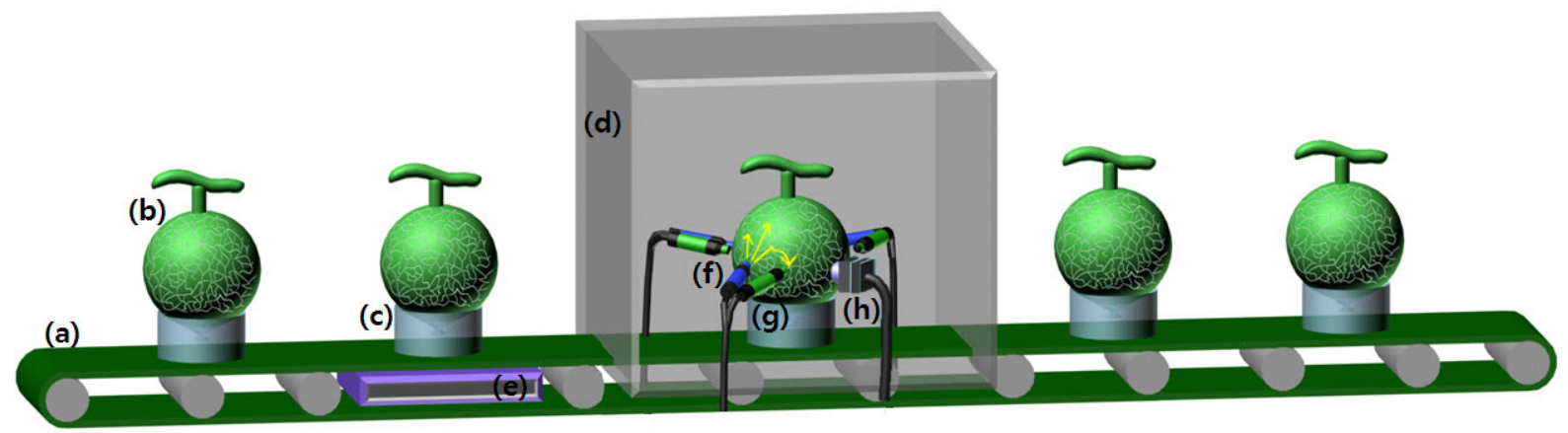

Figure 6. Schematic diagram of a fruit on-line grading machine: (a) conveyor belt, (b) fruit, (c) fruit hold tray, (d) darkroom chamber, (e) load cell, (f) light source, (g) spectrometer probe, and (h) camera.

prediction of SSC and firmness, respectively. In general, the $\mathrm{R}_{\mathrm{cv}}{ }^{2}$ values increase with the number of samples and the SEP values decrease. Those values begin being saturated at around 60 samples. The $\mathrm{R}_{\mathrm{cv}}{ }^{2}$ values at 60 samples are 0.711 (SEP $=0.93^{\circ} \mathrm{Brix}$ ) in experiment $1,0.713$ (SEP $=0.88$ ${ }^{\circ} \mathrm{Brix}$ ) in experiment 2, and 0.696 (SEP $=1.20^{\circ} \mathrm{Brix}$ ) in experiment 3 for the prediction of SSC and 0.750 (SEP= $3.30 \mathrm{~N})$ in experiment $1,0.414(\mathrm{SEP}=3.26 \mathrm{~N})$ in experiment 2, and $0.443(\mathrm{SEP}=4.53 \mathrm{~N})$ in experiment 3 for the prediction of firmness. Therefore, we believe that the calibration models need more than 60 samples to represent the variability of all samples with the measurement setup of this study.

\section{Melon fruit grading machine}

Although automated fruit sorting and packing houses have been built all over the country, most of harvested melons are still graded, sorted, and packed manually. In manual grading process, human graders first checks the color of fruit surface and the development of 'net', which is a network of suberized tissue. If the surface color of a fruit is not developed well or the pattern of net is not uniform, the fruit is offgraded. The fruits passed the appearance test are graded based on size or weight. For measurement of sweetness and firmness, a couple of samples are picked at every batch that is harvested in a same greenhouse. The SSC of the samples is measured by a refractometer in a destructive method. The firmness is estimated by pushing the bottom of a fruit.

To develop a fruit on-line grading machine by mimicking the human grading process, the grading machine should be equipped with a machine vision system for collecting the color of fruit surface and the characteristics of net, a load cell for measuring the weight of a fruit, and a spectrometer for estimating the SSC and firmness of a fruit in a nondestructive way. The schematic diagram of a fruit on-line grading machine is shown in Figure 6. A fruit is fed into the on-line grading machine by putting the fruit on a sample hold tray manually. The weight of the fruit in motion is measured by a load cell that is installed under conveyer belt. The fruit is moved into a darkroom chamber. The spectral data are collected by three sets of a light source and a spectrometer probe evenly distributed on equator of the fruit. Also, the surface color and the characteristics of net are measured by a machine vision system. The final grade of the fruit is determined by considering the weight, the characteristics of surface color and net, the SSC and firmness estimated by a spectrometer and then the fruit is sorted based on the grade and packed automatically.

\section{Conclusions}

In VIS/NIR spectroscopy, the effect of the configuration parameters in interactance mode on the estimation of SSC and firmness of muskmelon was investigated. The distance between a light source and a spectrometer probe affected the estimation of fruit attributes. At a $5 \mathrm{~cm}$ distance, the best results, the $\mathrm{R}_{\mathrm{cv}}{ }^{2}$ of 0.717 (SEP=1.16 ${ }^{\circ} \mathrm{Brix}$ ) and $0.504(\mathrm{SEP}=4.31 \mathrm{~N})$ for the estimation of SSC and firmness, respectively, were obtained. To fully represent the spectral characteristics of each fruit sample, at least 3 measurement points were required. The performance of the calibration models was various with the number of fruit samples. The performance began to be saturated at 60 fruit samples with the highest $\mathrm{R}_{\mathrm{cv}}{ }^{2}$ values of 0.713 (SEP= $\left.0.88^{\circ} \mathrm{Brix}\right)$ and $0.750(\mathrm{SEP}=3.30 \mathrm{~N})$ for the estimation of $\mathrm{SSC}$ and firmness, respectively. It could be concluded that the performance of the prediction models was quite dif- 
ferent according to the condition of interactance measurement setup. In designing a fruit grading machine with interactance configuration, the parameters for interactance measurement setup should be chosen carefully.

\section{Conflict of Interest}

No potential conflict of interest relevant to this article was reported.

\section{Acknowledgements}

This work was supported by Basic Science Research Program through the National Research Foundation of Korea (NRF) funded by the Ministry of Education, Science and Technology (MEST) (KRF-2007-314-D00343).

\section{References}

Nicolai, B. M., K. Beullens, E. Bobelyn, A. Peirs, W. Saeys, K. I. Theron and J. Lammertyn. 2007. Nondestructive measurement of fruit and vegetable quality by means of NIR spectroscopy: a review. Postharvest Biology and Technology 46: 99-118.

Penchaiya, P., E. Bobelyn, B. E. Verlinden, B. M. Nicolai and
W. Saeys. 2009. Non-destructive measurement of firmness and soluble solids content in bell pepper using NIR spectroscopy. Journal of Food Engineering 94: 267-273.

Schaare, P. N. and D. G. Fraser. 2000. Comparison of reflectance, interactance and transmission modes of visiblenear infrared spectroscopy for measuring internal properties of kiwifruit (Actinidia chinensis). Postharvest Biology and Technology 20:175-184.

Suh, S. R., K. H. Lee, S. H. Yu, H. S. Shin, Y. S. Choi and S. N. Yoo. 2012. A melon fruit grading machine using a miniature VIS/NIR spectrometer: 1. Calibration models for the prediction of soluble solids content and firmness. Journal of Biosystems Engineering 37(3):166-176.

Suh, S. R., K. H. Lee, S. H. Yu, S. N. Yoo and Y. S. Choi. 2011. Comparison of performance of measuring of VIS/NIR spectroscopic spectrum to predict soluble solids content and 'Shingo' pear. Journal of Biosystems Engineering 36(2):130-139 (In Korean, with English abstract).

Sun, T., K. Huang, H. Xu and Y. Ying. 2010. Research advances in nondestructive determination of internal quality in watermelon/melon: A review. Journal of Food Engineering 100:569-577.

Zude, M., B. Herold, J. M. Roger, V. Bellon-Maurel and S. Landahl. 2006. Non-destructive tests on the prediction of apple fruit flesh firmness and soluble solids content on tree and in shelf life. Journal of Food Engineering 77:254-260. 